\title{
The Sleep-E Study: An on-going cross-sectional study investigating associations of sleep quality and cardio-metabolic risk factors
}

\author{
H.K. Al Khatib, S.K. Kfoury, J. Mehat, G.K. Pot*, S.V. Harding* and J. Darzi* \\ Diabetes \& Nutritional Sciences Division, Faculty of Life Sciences \& Medicine, King's College London, Franklin \\ Wilkins Building, 150 Stamford Street, London, SE1 9NH
}

Short sleep duration ( $\leqslant 5-6 \mathrm{~h} /$ night) has been associated with an increased risk of weight gain $(1,2)$ and non-communicable disease $(3)$. Recently, through a systematic review and meta-analysis, we identified that short sleep duration may induce positive energy balance and have implications on weight gain over the long term (Al Khatib et al, unpublished data). Quality of sleep, opposed to its duration, has not been extensively reported in the context of the impact on disease risk. While self-reported measures of sleep quality have been linked to markers of adiposity and insulin resistance (4), there are no reports of objective measures of sleep quality in free-living conditions and cardio-metabolic disease risk. The Sleep-E Study is an on-going, cross-sectional study assessing associations between objective measures of sleep and cardio-metabolic risk factors in healthy adults, aged $18-55$ years $(\mathrm{N}=29 ; 12 \mathrm{M} / 17 \mathrm{~F})$. It is being conducted in accordance with the Declaration of Helsinki. Sleep parameters were assessed using the MotionWatch8 (CamNtech Ltd, Cambridge, UK) for six continuous nights, using sleep latency (time taken to transition from wakefulness to sleep) and sleep efficiency (actual sleep time expressed as a percentage of time in bed) as indicators of sleep quality. Fasting blood samples, blood pressure and anthropometric measurements were taken on study day 1. Correlations between sleep quality indicators and cardiometabolic risk factors, adjusted for age, sex and BMI, were investigated.

\begin{tabular}{|c|c|c|c|c|c|c|}
\hline \multirow{2}{*}{$\begin{array}{l}\text { Cardio-metabolic } \\
\text { Risk Factor }\end{array}$} & \multicolumn{2}{|c|}{ Sleep efficiency $(\%)$} & \multicolumn{2}{|c|}{ Sleep latency (hh:mm) } & \multicolumn{2}{|c|}{ Sleep duration (hh:mm) } \\
\hline & $\mathrm{r}$ & $P$ value & $\mathrm{r}$ & $P$ value & $\mathrm{r}$ & $P$ value \\
\hline $\mathrm{WC}, \mathrm{cm}$ & -0.208 & $0 \cdot 31$ & $0 \cdot 372$ & 0.06 & -0.076 & 0.71 \\
\hline Body Fat, \% & -0.336 & 0.09 & $0 \cdot 320$ & $0 \cdot 11$ & $0 \cdot 167$ & 0.42 \\
\hline $\mathrm{SBP}, \mathrm{mm} \mathrm{Hg}$ & 0.229 & $0 \cdot 26$ & $-0 \cdot 338$ & 0.09 & $-0 \cdot 157$ & 0.44 \\
\hline $\mathrm{DBP}, \mathrm{mm} \mathrm{Hg}$ & -0.316 & $0 \cdot 12$ & 0.363 & 0.07 & -0.087 & 0.67 \\
\hline Glucose, $\mathrm{mmol} / \mathrm{L}$ & 0.029 & $0 \cdot 89$ & -0.156 & $0 \cdot 45$ & $0 \cdot 294$ & $0 \cdot 15$ \\
\hline $\mathrm{TG}, \mathrm{mmol} / \mathrm{L}$ & -0.763 & $<0.001$ & $\mathbf{0 . 5 7 7}$ & 0.002 & $-0 \cdot 070$ & 0.74 \\
\hline $\mathrm{NEFA}, \mathrm{mmol} / \mathrm{L}$ & $-0 \cdot 013$ & 0.95 & $0 \cdot 271$ & $0 \cdot 18$ & $-0 \cdot 129$ & 0.53 \\
\hline $\mathrm{TC}, \mathrm{mmol} / \mathrm{L}$ & -0.259 & $0 \cdot 20$ & $0 \cdot 186$ & $0 \cdot 36$ & 0.219 & 0.28 \\
\hline $\mathrm{LDL}, \mathrm{mmol} / \mathrm{L}$ & $-0 \cdot 180$ & 0.38 & $0 \cdot 150$ & 0.47 & 0.118 & 0.57 \\
\hline $\mathrm{HDL}, \mathrm{mmol} / \mathrm{L}$ & 0.112 & 0.59 & $0 \cdot 181$ & 0.11 & 0.336 & 0.09 \\
\hline TC:HDL ratio & -0.427 & 0.03 & 0.436 & 0.03 & $-0 \cdot 143$ & 0.49 \\
\hline
\end{tabular}

DBP, diastolic blood pressure; HDL, high density lipoprotein; LDL, low density lipoprotein cholesterol; NEFA, non-esterified fatty acids; r, Pearson partial correlation coefficient; SBP, seated systolic blood pressure; TG, triglycerides; TC, total cholesterol; WC, waist circumference.

These data show a strong negative correlation between sleep efficiency and TG concentrations and a moderate positive correlation with TC:HDL ratios. Similarly, sleep latency was moderately and positively correlated with both triglyceride concentrations and TC: HDL ratios. Sleep duration did not correlate with any risk factors. This cross-sectional data suggests that compromised sleep quality is linked to lipid metabolism. Sleep quality, independent of sleep duration, may be a potential, novel target for cardio-metabolic disease prevention. This cross-sectional study demonstrates the need for intervention studies assessing the relationship between sleep quality and cardio-metabolic risk.

1. Cappuccio FP, Taggart FM, Kandala NB, et al. (2008) Sleep 31, 619-26.

2. Patel SR \& Hu FB (2008) Obesity 16, 643-53.

3. Cappuccio FP, Cooper D, D'Elia L, et al. (2011) Eur Heart J 32, 1484-92.

4. Jennings JR, Muldoon MF, Hall M, et al. (2007) Sleep 30, 219-23.

*all contributed equally. 